\title{
EL POSICIONAMIENTO DE LOS MEDIOS DE COMUNICACIÓN ENTRE LA COMUNIDAD MÉDICA ESPECIALIZADA EN ESTUDIOS GENÉTICOS
}

\author{
Delia Outomuro, Lorena Mariel Mirabile y Natalia Cosmai*
}

\begin{abstract}
Resumen: Este ensayo forma parte del proyecto de investigación "La representación social de la investigación genética entre la comunidad científica y la población urbana de Buenos Aires. Su influencia en el diálogo bioético", enmarcado en la Programación Científica 2004-2008 de la Universidad de Buenos Aires, que tiene como objetivo indagar sobre la construcción del imaginario social en relación con los avances científico-tecnológicos en torno a la genética-considerando la selección de los medios de comunicación que transmiten la información a la población general y a la comunidad médica- y analizar cómo esa construcción determina políticas sociales de salud y otras.
\end{abstract}

Palabras clave: medios de comunicación, investigación, genética, población médica, imaginario social, prejuicio

\section{MEDIA POSITIONING OF SPECIALIZED MEDICAL COMMUNITY IN GENETIC STUDIES}

Abstract: This paper forms part of the research project: "Social representation of genetic research among the scientific community and the urban population in Buenos Aires. Its influence in the bioethics dialogue," framed in the scientific program of the University of Buenos Aires for 2004-2008. Its objective is to inquire about the social imagery construct connected to technological and scientific achievements in genetics - considering the selection done by the media when informing the general population and the medical community- and to analyze how this construct determines health care social policies and other policies as well.

Key words: media, research, genetics, medical community, social imagery, prejudice

\section{O POSICIONAMENTO DOS MEIOS DE COMUNICAÇÃO ENTRE A COMUNIDADE MÉDICA ESPECIALIZADA NOS ESTUDOS GENÉTICOS}

Resumo: Este artigo é parte de o projeto de pesquisa "A representação social da pesquisa genética entre a comunidade científica e a população urbana de Buenos Aires. Sua influência no diálogo bioético”, situado na Programação Científica 2004-2008 da Universidade de Buenos Aires. Esta projeto tem como objetivo indagar sobre a construçáo do imaginário social na relação com os avanços científico-tencológicos em torno da genética, considerando a seleção dos meios de comunicação que transmitem a informação à populaçáo geral e a comunidade médica, bem como analisar como esta construçâo determina políticas sociais de saúde e outras.

Palavras-chave: meios de comunicação, pesquisa genética, população médica, imaginário social, preconceito

* Unidad Académica de Bioética. Departamento de Humanidades Médicas. Facultad de Medicina. Universidad de Buenos Aires, Argentina Correspondencia: doutomuro@fmed.uba.ar 


\section{Introducción ${ }^{1}$}

"Clonación”, "terapia génica", "diagnóstico genético", "proyecto genoma humano", "farmacogenética”, y la lista podría continuar. Estos tópicos inundan las páginas de diarios y revistas y son presentados en programas "científico" por radio y televisión. Pero, ¿qué información transmiten los medios? ¿Cómo se transmite? ¿Cuán fiable es? ¿Qué intereses están en juego? Indagar sobre estas y otras cuestiones similares no es tarea ociosa, en la medida en que se reconoce la influencia de los medios sobre la ciudadanía, la que, a su vez, tiene la responsabilidad de legitimar o no las prácticas mencionadas.

La valoración de los medios de comunicación como formadores de opinión pública y fuentes de información no neutral ha sido tema de intensa reflexión en las últimas décadas. La elaboración del informe $M c$ Bride (UNESCO) expuso el Nuevo Orden Mundial en Información y Comunicaciones (NOMIC) y denunció el flujo desequilibrado cualicuantitativo de la información circulante en el mundo, en función de los intereses de los países centrales que monopolizan y mercantilizan la comunicación $(1,2)$. Los trabajos en este campo desplazaron el eje inicial de análisis desde la instancia de recepción y el estudio de la estructura del mensaje hacia las de emisión de los discursos absorbidos por la comunidad(3).

El NOMIC puso de manifiesto el colonialismo informativo o comunicacional y evidenció que el libre flujo de información no debía equipararse con flujo libre y equilibrado(2,4). Asimismo, rejerarquizó la concepción de la comunicación como servicio público, diferenciándolo del servicio de interés público, y redimensionó la información como bien social en vez de considerarla como mercancía pasible de ser afectada por las leyes del mercado(5).

Nacía así, en la década de los 70, sobre el escenario creado por la elaboración del Informe $M c$ Bride, la

1 Este trabajo se realizó en el marco de la programación científica 20042008 de la Universidad de Buenos Aires, Proyecto UBACYT M088 "La representación social de la investigacion genética entre la comunidad científica y la población urbana de Buenos Aires. Su influencia en el diálogo bioético", vinculado con el proyecto \#de-FG02-02ER63435 US. Department of Energy "Representation of genomics research among Latin American laymen and bioethicists. A inquiry into the migration of knowledge and its impact on underdeveloped communities", llevado a cabo en el Centro Interdisciplinario de Estudios en Bioética, Universidad de Chile, bajo la dirección de Fernando Lolas Stepke. llamada "sociopolítica de la comunicación"(3). Bajo este panorama, los medios de comunicación masiva se consideran vehículos de expresión simbólica y cultural de las clases dominantes. Ellas mediatizan bajo esta forma los valores inherentes a la industrialización y a la tercera fase del capitalismo, conocida como "fase transnacional" (6-8). Desde esta óptica, los medios se consideran como una de las variables más importantes y con más influencia sobre la opinión pública. Son las voces más reconocidas en la polifonía discursiva $(9,10)$.

En América Latina la "democracia comunicacional" es vista como una técnica o un método en lugar de ser el objetivo; lo que se quiere destacar con esta frase es que en la sociedad neoliberal e informacional se han conformado dos sectores de la población: uno con acceso a la información y que puede elegir cómo, cuándo y cuánto desea adquirir de la oferta expuesta, y otro inserto también en una sociedad de consumo, pero que carece de información y, por tanto, sus elecciones son dirigidas por el mercado, emergiendo de allí la fortaleza de los monopolios. En los países periféricos se generan los multimedios privados, nacionales e internacionales, que monopolizan la información en pocas manos hegemónico-dominantes. Estos conglomerados multimediáticos concentran y manejan la información en una selecta minoría(11-15).

Son múltiples los factores que intervienen en la conformación de la opinión pública en torno al tema de la genética, pero los medios de comunicación constituyen una variable relevante y fundamental. Son ellos los que ponen la información al alcance de la comunidad no especializada y, como venimos diciendo, lo que se comunica está fuertemente cargado de intereses partidarios.

En este trabajo partimos del supuesto de que la comunidad de médicos genetistas conoce "la verdad" sobre estos temas. Como se verá, esa verdad es entendida como correspondencia entre lenguaje y realidad; es una verdad como aletheia (develada por desocultamiento) y es la comunidad médica (o científica) quien tiene el poder y el deber de descubrirla. Esa "verdad" constituye la regla y el punto de referencia con el que los médicos valoran la información (el qué y el cómo) transmitida por los medios. Dentro de este marco teórico, indagamos qué piensan los médicos genetistas acerca del manejo de la información sobre genética en los medios masivos de comunicación. 


\section{Diseño de investigación, materiales e instrumentos. Población y muestra}

Tomando en consideración la naturaleza psicosocial de lo analizado, se decidió la utilización de un enfoque tanto cuantitativo como cualitativo. Dentro del primero, se optó por la encuesta con prevalencia de preguntas abiertas (asociación de palabras, frases incompletas, diferencial semántico, etc.), pero, debido a que los resultados esperados no son objetivamente mensurables y no pueden sintetizarse en forma numérica, se utilizó especialmente el enfoque cualitativo en el análisis e interpretación de los datos. Dentro del segundo enfoque, se eligió como técnica de recolección de datos la de entrevistas semiestructuradas. Si bien se utiliza una guía de preguntas, su principal objetivo es dejar al entrevistado la posibilidad de expresarse libremente y no estructurar su discurso.

La utilización de estas entrevistas permitió acceder, mediante preguntas abiertas, a la construcción social del imaginario de la comunidad de investigadores médicos relacionados con lo genético, y conocer cómo esta comunidad se autopercibe frente al entorno contingente. Además, reveló índices de hostilidad o afectividad frente a su propia reacción. Finalmente, esta técnica informó acerca de la presencia o ausencia de reflexión sobre el conglomerado mediático, y/o de prejuicios relacionados con los canales de información $\mathrm{y}$ su función o funcionalidad social.

Es destacable la utilización del sistema de calificación "diferencial semántico" (semantic differential) o escala bipolar de conceptos antagónicos en duplas (blanco-negro, bueno-malo, metal-madera, cálido-frío, duro-blando, etc.), utilizado en éste y otros trabajos de investigación. Este método de visualización, desarrollado por Charles E. Osgood a fines de los 50, cumple el objetivo de representar gráficamente las diferentes connotaciones asociadas con una palabra en diferentes individuos.

La selección de las unidades observacionales se realizó de manera no probabilística, por voluntad del investigador. Se tomó como muestra de la población general de médicos investigadores de temas genéticos a un conjunto de 18 personas (7 hombres y 11 mujeres) de entre 30 y 60 años. Los entrevistados fueron informados sobre los alcances de la investigación, el anonimato de los informes y el resguardo de la confidencialidad, y consintieron participar en el proyecto.
Es importante destacar que las técnicas cualitativas de recolección de datos son más fructíferas que las cuantitativas cuando de posicionamientos se trata. "Posicionar" significa ubicar contextualmente, prejuzgar estableciendo jerarquías, órdenes y prioridades mentales. La naturaleza de lo que se busca conocer responde a un cuestionamiento más cualitativo que cuantitativo. Por eso los resultados del primer análisis pueden variar cuando se indaga más profundamente mediante una técnica cualitativa, como es la entrevista en profundidad. La repregunta, la indagación tangencial, la libre asociación de palabras, entre otras formas de operacionalización, revelan mejor ese "prejuicio" que la calculada respuesta estandarizada de un cuestionario predeterminado.

\section{Resultados}

Las tablas que se presentan a continuación responden a resultados del enfoque cuantitativo. La evaluación cualitativa se informa en la discusión, en la que se transcriben textualmente aquellas apreciaciones que se consideraron relevantes.

Si bien en una primera aproximación pareciera que los resultados numéricos no tienen relación con la valoración cualitativa plasmada en la discusión, los datos del enfoque cuantitativo se completan con el análisis cualitativo. Muchas veces el plano de lo mensurable sólo permite una visualización del tema; lo cualitativo funciona mejor en la focalización y en la profundidad de análisis. Esto proporciona la ventaja de explotar temas no contemplados inicialmente y ahondar en algunos de los contemplados superficialmente.

La tabla 1 presenta la autoevaluación de los médicos genetistas con relación a su conocimiento sobre: proyecto genoma humano, aplicaciones de dicho proyecto en medicina, etiología genética de las enfermedades, pruebas de diagnóstico genético, clonación y medicina en general.

A grandes rasgos podemos concluir que las mujeres son más cautelosas al hablar sobre "su conocimiento médico". Si bien "bastante" fue la respuesta más elegida, la opción "regular" está presente en todos los items evaluados. Los hombres se refieren a sí mismos como conocedores del tema, como más instruidos e ilustrados. 
Tabla 1: Autoevaluación de los médicos con respecto a temas genéticos: ¿Cuánto creen saber los médicos genetistas sobre...?

\begin{tabular}{|l|c|c|c|c|c|c|c|c|c|c|c|c|}
\hline \multicolumn{2}{|c|}{$\begin{array}{c}\text { Proyecto genoma } \\
\text { humano }\end{array}$} & $\begin{array}{c}\text { Aplicaciones del } \\
\text { proyecto }\end{array}$ & $\begin{array}{c}\text { Enfermedades } \\
\text { genéticas }\end{array}$ & \multicolumn{2}{c|}{$\begin{array}{c}\text { Test o pruebas } \\
\text { genéticas }\end{array}$} & \multicolumn{3}{c|}{ Clonación } & \multicolumn{2}{c|}{ Medicina } \\
\hline & $\mathrm{H}$ & $\mathrm{M}$ & $\mathrm{H}$ & $\mathrm{M}$ & $\mathrm{H}$ & $\mathrm{M}$ & $\mathrm{H}$ & $\mathrm{M}$ & $\mathrm{H}$ & $\mathrm{M}$ & $\mathrm{H}$ & $\mathrm{M}$ \\
\hline Mucho & 1 & 1 & 1 & 1 & 2 & 1 & 0 & 1 & 0 & 2 & 2 & 2 \\
\hline Bastante & 5 & 3 & 5 & 4 & 5 & 6 & 7 & 5 & 6 & 2 & 5 & 4 \\
\hline Regular & 0 & 4 & 1 & 4 & 0 & 4 & 0 & 5 & 1 & 4 & 0 & 5 \\
\hline Poco & 1 & 3 & 0 & 0 & 0 & 0 & 0 & 0 & 0 & 3 & 0 & 0 \\
\hline Nada & 0 & 0 & 0 & 2 & 0 & 0 & 0 & 0 & 0 & 0 & 0 & 0 \\
\hline TOTALES & 7 & 11 & 7 & 11 & 7 & 11 & 7 & 11 & 7 & 11 & 7 & 11 \\
\hline
\end{tabular}

La tabla 2 muestra una escala de valores de 1 a 10 donde 1 es el desacuerdo total y 10 es la coincidencia plena. Durante la encuesta se comunicó a los encuestados diversas opiniones vertidas por coetáneos entre las cuales figuraban dos enunciados que se resumían en "los medios de comunicación están bien informados" y "los diarios, la televisión y la radio no ocultan información”. Los resultados muestran que, en la mayoría de los casos, los médicos y médicas están muy en desacuerdo con las opiniones enunciadas.

Tabla 2: Visión médica del tratamiento de los temas médicos en los medios de comunicación

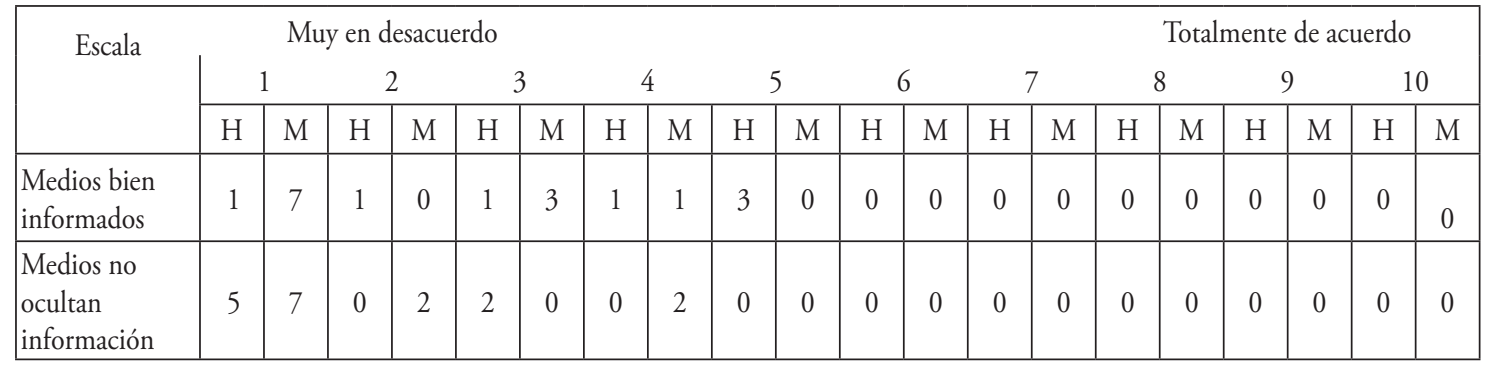

Es particularmente paradigmática la actitud de los encuestados frente al enunciado "los medios no ocultan información". Aquí es importante recalcar los límites del alcance cuantitativo de análisis. En una primera aproximación sería correcto concluir que la comunidad analizada comprende que los medios de comunicación ocultan adrede y con intenciones. Sin embargo, durante las entrevistas en profundidad, surgió la idea de que "ocultar" estaría más relacionado con "no emitir información" -por ejemplo, por cuestiones de rating- que como modo racional y sistematizado de moldear mentes en función de los intereses de las clases hegemónicas.

En la tabla 3, el diferencial semántico nos resultó de gran utilidad para indagar el pensamiento del encuestado. Logramos focalizar y entender el posicionamiento de los diferentes medios de comunicación, estableciendo diferencias de significado/denotación y sentido/connotación. Aquí se buscó acceder al segundo binomio a partir del primero. En los casos de "verdadero-falso" o "bueno-malo" la relación denotación/connotación es obvia; pero en el caso de otros binomios se requiere un esfuerzo extra y una asignación de carga significante previa a la respuesta del entrevistado por parte del investigador. Es así como "blanco" implica "puro", "sin intención”, "natural”, "sano", "honesto" y "metal” connota "limpio", "vanguardista”, "estático" "futurista", "serio", "respetable", "inenjuiciable". Los resultados presentados en esta tabla se comentan en la discusión. 
Tabla 3: Apreciaciones de la comunidad médica relacionada con la genética sobre los medios de comunicación. ¿Qué significan los diferentes medios?

\begin{tabular}{|c|c|c|c|c|c|c|}
\hline \multicolumn{7}{|c|}{ TELEVISIÓN } \\
\hline MALO & 0 & 10 & 6 & 2 & 0 & BUENO \\
\hline FRÍO & 0 & 7 & 7 & 4 & 0 & CÁLIDO \\
\hline DURO & 0 & 5 & 5 & 6 & 2 & BLANDO \\
\hline CUADRADO & 1 & 7 & 5 & 3 & 2 & REDONDO \\
\hline FALSO & 5 & 5 & 7 & 0 & 1 & VERDADERO \\
\hline METAL & 4 & 2 & 7 & 2 & 3 & MADERA \\
\hline NEGRO & 6 & 6 & 6 & 0 & 0 & BLANCO \\
\hline \multicolumn{7}{|c|}{ DIARIO } \\
\hline MALO & 2 & 1 & 2 & 6 & 7 & BUENO \\
\hline FRÍO & 3 & 2 & 6 & 3 & 4 & CÁLIDO \\
\hline DURO & 1 & 2 & 8 & 5 & 2 & BLANDO \\
\hline CUADRADO & 3 & 4 & 6 & 3 & 2 & REDONDO \\
\hline FALSO & 1 & 4 & 12 & 1 & 0 & VERDADERO \\
\hline METAL & 1 & 2 & 11 & 3 & 1 & MADERA \\
\hline NEGRO & 2 & 3 & 11 & 2 & 0 & BLANCO \\
\hline \multicolumn{7}{|c|}{ RADIO } \\
\hline MALO & 0 & 3 & 8 & 4 & 3 & BUENO \\
\hline FRÍO & 0 & 1 & 7 & 7 & 3 & CÁLIDO \\
\hline DURO & 1 & 1 & 10 & 5 & 1 & BLANDO \\
\hline CUADRADO & 0 & 4 & 7 & 7 & 0 & REDONDO \\
\hline FALSO & 1 & 4 & 8 & 5 & 0 & VERDADERO \\
\hline METAL & 1 & 3 & 7 & 6 & 1 & MADERA \\
\hline NEGRO & 2 & 0 & 12 & 4 & 0 & BLANCO \\
\hline \multicolumn{7}{|c|}{ REVISTA } \\
\hline MALO & 0 & 3 & 7 & 5 & 3 & BUENO \\
\hline FRÍO & 2 & 3 & 5 & 4 & 4 & CÁLIDO \\
\hline DURO & 1 & 1 & 6 & 6 & 4 & BLANDO \\
\hline CUADRADO & 3 & 3 & 7 & 3 & 2 & REDONDO \\
\hline FALSO & 2 & 4 & 10 & 2 & 0 & VERDADERO \\
\hline METAL & 1 & 2 & 6 & 5 & 4 & MADERA \\
\hline NEGRO & 1 & 1 & 10 & 5 & 1 & BLANCO \\
\hline \multicolumn{7}{|c|}{ REVISTA ESPECIALIZADA } \\
\hline MALO & 0 & 0 & 5 & 5 & 8 & BUENO \\
\hline FRÍO & 5 & 5 & 6 & 1 & 1 & CÁLIDO \\
\hline DURO & 6 & 10 & 0 & 2 & 0 & BLANDO \\
\hline CUADRADO & 4 & 3 & 6 & 3 & 2 & REDONDO \\
\hline FALSO & 0 & 0 & 6 & 7 & 5 & VERDADERO \\
\hline METAL & 2 & 6 & 10 & 0 & 0 & MADERA \\
\hline NEGRO & 0 & 1 & 9 & 6 & 2 & BLANCO \\
\hline
\end{tabular}

\section{Discusión}

Podemos adelantar que el posicionamiento -ese prejuicio que se tiene sobre "algo"- de los medios de comunicación masiva entre la comunidad médica argentina más ligada a la investigación genética es "bipartidario" y existe en torno a dos posiciones claramente marcadas y dicotómicas. Ambas tienden a entender a los medios como fuentes de información, como canales 
de comunicación masiva. La diferencia de posturas radica en la forma en que se percibe aquello que es encomendado a los medios (por ejemplo ser objetivos, describir neutralmente, etc.) más que en la conceptualización del término "medio de comunicación". Es decir, ambas aproximaciones al concepto "medio de comunicación" tienen en común la "descripción" del objeto, pero mientras una se limita a describirlo, la otra además lo juzga.

La primera posición entiende a "los medios de comunicación" como netos canales de información, cualquiera sea éste. Ellos son caracterizados como trasmisores de conocimiento, se los enumera y reconoce pero no se reflexiona acerca de su funcionalidad. Tampoco se abre juicio sobre cómo informan o por qué lo hacen. Los representantes de esta posición limitan su entendimiento a la descripción lineal de lo que se conoce como "canal masivo de información". Los medios se describen como un "instrumento para que la población se informe" o bien "toda forma escrita, visual o audiovisual que se utiliza para la trasmisión de información" sin detenerse en la especulación acerca de quién los usa o maneja y con qué fines.

Oscilando hasta la postura contraria, encontramos una mirada un tanto más reflexiva de crítica espontánea. Los medios son percibidos como canales de comunicación que "deberían estar bien informados", o ser "supervisados" (¿por quién?). "Deberían ser buenos difusores de información certera" o bien son "sistemas masivos de información (...) que suministran datos a la población con ciertas subjetividades y que no son independientes de ideologías partidarias". Estas citas referidas por los entrevistados demuestran una intención de cálculo y cavilación en torno al tema. Sin embargo, se mantienen en el nivel de crítica o reclamo (deberían ser esto o aquello) sin cuestionar el por qué.

Este posicionamiento contempla la desinformación como característica fundamental de los medios pero no especula acerca de su causa. Deja librado al azar o al mero fin de informar "lo que sea" sin indagar más fuertemente sobre esta temática que conlleva el hecho de estar desinformados. "Desinformados" implica, desde esta postura, "carentes de conocimiento" y no "ocultamiento por conveniencia". Los medios no saben, "no están bien informados", pero no ocultan adrede. En muy pocos casos se detectan pensamientos como "no son independientes de ideologías partidarias".
Es importante destacar la existencia de reflexión sobre los medios de comunicación masiva en este segundo posicionamiento de la población estudiada, si bien esto no sucede espontáneamente en la mayoría de los casos. Los medios son reconocidos, enumerados y comprendidos como "formas de interrelación comunitaria que inciden en la formación de una opinión". Son susceptibles de juicio ético a la vez que descriptivo. No son banalizados ni desmerecidos. Se les reconoce la capacidad de generar opinión y "crear expectativas". Si bien no hay credibilidad en los medios de comunicación, se les considera interlocutores válidos, ya que desmerecer algo implica tenerlo en cuenta.

En síntesis, lo que no se reconoce es la "sociopolítica de la comunicación”. Como se indicó en la introducción, la forma en que se construye conocimiento y cómo éste se vuelve significativo para la sociedad en su conjunto está fuertemente relacionada con la condición de periferia o de centro que tienen los países del mundo. Las relaciones de los países entre sí dependen de su condición productiva y de su capacidad resolutiva. Hoy, la capacidad de producción -no sólo de productos manufactureros sino también de conocimiento científico y tecnológico- descansa en los países de Europa occidental, América septentrional y Asia. Esta capacidad les ha otorgado el título de países desarrollados y con ello adquieren el derecho a generar políticas de acción y de reflexión, decisiones económicas y éticas. Por su parte, los países subdesarrollados asumen el papel de consumidores pasivos. No obstante, si bien ellos no pueden reproducir los aspectos técnicos de la investigación genética producida en los países centrales, es menester que, debido al proceso de globalización, se preocupen por su influencia.

Por otro lado, se reconoce la existencia de una "verdad científica" que merece ser develada o descubierta por la comunidad médica (o científica). Esta verdad no es entonces construida sino des-ocultada. Mucho menos se la comprende como histórica o conveniente. No hay indicios de un pensamiento que reflexione acerca de la "real existencia" de la verdad o de su comprensión por parte del género humano. Es aletheia y será descubierta. Este pensamiento está en consonancia con una filosofía positivista, fuertemente arraigada en la comunidad científica en general y entre los médicos en $\operatorname{particular}(16)$.

El positivismo comulga con el realismo al afirmar que el mundo exterior existe independientemente del sujeto y 
puede llegar a conocerse tal como es(17). Los médicos entrevistados defienden implícitamente un realismo metafísico que conlleva la aceptación de tres tesis: independencia y totalidad fija de la realidad y verdad como correspondencia. En otras palabras: los objetos del mundo existen independientemente de nuestro conocimiento -mente, lenguaje, esquemas conceptuales o representaciones- y constituyen una totalidad fija; por lo tanto, hay una única descripción verdadera y completa del mundo, cuya verdad consiste en la correspondencia entre las palabras y los objetos(18).

Los médicos entrevistados consideran que el conocimiento se adquiere de un modo contemplativo y no activamente como corresponde a su condición de producto social. El conocimiento es una mera copia o reflejo de la realidad, no se construye a partir de conocimientos previos y de recursos culturales preexistentes. Su aceptación y mantenimiento tiene que ver sólo con la cuestión de cómo ese conocimiento se relaciona con la realidad. No se percibe su relación con los intereses y fines a alcanzar por una comunidad determinada(19). Por lo tanto, la no visualización del conocimiento como un hecho social desecha la posibilidad de adoptar una concepción pragmática de la verdad, según la cual el conocimiento verdadero es aquello que los actores sociales toman como tal y, por tanto, la verdad queda determinada por las metas y los intereses de cada comunidad histórica y culturalmente situada(20).

Si tomamos en consideración que, en la mayoría de los casos, los investigadores se reconocen como instruidos y competentes en temas de genética, es de suponer que ellos deberían ser utilizados por los medios como "reales" y "certeras" fuentes de información.

Los medios tampoco son percibidos como empresas con fines de lucro. No hay conciencia del conglomerado mediático ni rastros de un pensamiento crítico al respecto. La radio, la televisión, el diario y la revista son entes que corren por cuerdas separadas y, por ello, se les asigna un sentido distinto. Esta significación diferencial se manifiesta más claramente cuando se los compara con medios especializados, como las revistas científicas.

Como era de esperar, estas últimas poseen un estatus ontológico disímil respecto del resto de los medios. Mientras éstos estimulan la opinión, el juicio y el dictamen; aquéllas generan respeto. Los medios "tradicionales" originan sentimientos; las revistas científicas predisponen el pensamiento. El sentido generado por estas últimas las identifica y discrimina del resto: no son medios de comunicación y no generan sentido crítico. Muy por el contrario, son objetivas, calculadas, lógicas y racionales $y$, por lo tanto, no pueden ser pasibles de juicio alguno. Están del lado de "lo verdadero" y "lo bueno". Según la comunidad médica, no marcan tendencias de pensamiento y no se les asigna la capacidad de generar opinión. Se esgrimen como lugares de información veraz y concreta.

La televisión encabeza la lista de "los medios malos", aquellos menos objetivos, menos racionales, mal informados. La televisión es "falsa", "fría" y "negra". Luego se nombran los diarios, que tienden a ser construidos -al igual que la radio- como más "cálidos", más "personales", más amigables. Por último, las revistas no especializadas son menos "entendidas" y no estimulan ningún pensamiento crítico significativo. Podrían ser contempladas como inofensivas.

Si bien hay ciertas diferencias entre los grupos, éstas son mínimas: ni la edad, ni el género son factores determinantes en la construcción del posicionamiento mediático. No hay huellas significantes que merezcan asociar las variables "edad" y "género" con las diferentes cosmovisiones.

\section{Conclusiones}

1) La comunidad médica relacionada con temas genéticos reconoce la influencia de los medios de comunicación en la conformación de la opinión pública, pues los concibe no sólo como "canales" de información sino también como "fuentes".

2) Se identificaron dos grupos de pensamiento. Si bien ambos posicionamientos tienen en común la "descripción” del objeto, un grupo se limita a describir el concepto "medio de comunicación" mientras que la otra aproximación además lo juzga.

3) Este segundo posicionamiento, que transciende el plano meramente descriptivo, entiende que los medios están desinformados y que, por tanto, son falaces como fuente de conocimiento.

4) La reflexión, sin embargo, no va más allá; se mantiene en el nivel de crítica o reclamo epistémico, no se pregunta por qué, no indaga sobre posibles intereses que puedan explicar la desinformación.

5) El desconocimiento de una sociopolítica de la comunicación comulga con una visión positivista 
de la ciencia y la medicina: verdad como correspondencia y como aletheia, realismo metafísico y hegemonía de saber científico sobre otras formas de conocimiento son supuestos aceptados por la población estudiada.
6) Se percibieron disparidades entre los posicionamientos con relación a los diferentes soportes materiales, y la televisión encabezó la lista de los medios considerados menos objetivos y menos racionales.

7) No se apreciaron huellas significantes asociadas a las variables edad y género.

\section{Referencias}

1. Argumedo A. Los laberintos de la crisis. América Latina: poder transnacional y comunicaciones. Montevideo, Buenos Aires: Instituto Latinoamericano de Estudios Transnacionales, Puntosur Editores: 1987.

2. Hamelink C. Nuevos desequilibrios en el flujo internacional de las comunicaciones. Voces y Culturas 1991; 2/3.

3. Pasquali A. Comunicación y cultura de masas. I. Caracas: Monte Ávila; 1964.

4. Graziano M. Política o ley: debate sobre el debate. Revista Espacios UBA 1986.

5. Roncagliolo R. Comunicación y democracia en el debate internacional. Revista Chasqui 1983; 7.

6. Oszlak O, O’Donnell G. Estado y políticas estatales en América Latina: hacia una estrategia de investigación. Revista Venezolana de Desarrollo Adminsitrativo 1982; 1.

7. Boron A. La sociedad civil después del diluvio neoliberal. En: Sader E, Gentili P, (comps.) La trama del neoliberalismo. Mercado, crisis y exclusión social. Buenos Aires: CLACSO/EUDEBA; 2003.

8. García Delgado D. Estado y Sociedad: Buenos Aires: Editorial Tesis; 1994.

9. Hamelink C. Hacia una Autonomía Cultural en las Comunicaciones Mundiales. Caps. I y III. Buenos Aires: Ed. Paulinas; 1985.

10. Rossi D. La radiodifusión en la Argentina neoliberal. Buenos Aires: CECSO mimeo; 1995.

11. Albornoz L, Hernández P, Mastrini G, Postolski G. "Al fin solos", el nuevo escenario de las comunicaciones en la Argentina. En: Albornoz L, (comp.) Al fin solos... la nueva televisión del Mercosur. Buenos Aires: Ed. Ciccus/La Crujía; 2000.

12. Anderson P. Más allá del neoliberalismo: Lecciones para la izquierda. En: Sader E, Gentilli P, (comps.) La trama del neoliberalismo. Mercado, crisis y exclusión social. Buenos Aires: CLACSO-EUDEBA; 1999: 143-147.

13. Mastrini G, Becerra M. Concentración de medios. Buenos Aires: UBA (mimeo); 2003.

14. Mastrini G, De Charras D. Veinte años no es nada: del NOMIC a la CMSI. Ponencia al Congreso IAMCR 2004, Porto Alegre, Brasil.

15. Graziano M. Los dueños de la TV argentina. Revista Comunicación y Cultura 1974; 3.

16. Kohn Loncarica AG, Outomuro D. El poder de la medicina y el derecho a la información del paciente. En: Aberastury P, Amaya JA, Barker RS, et al. Poder político y libertad de expresión. Buenos Aires: Ed. Abeledo Perrot; 2001.

17. Pérez Ransanz A. Kubn y el Cambio Científico. México: FCE; 1999.

18. Barnes B. El problema del conocimiento. En: Olivé L, (comp.) La explicación social del conocimiento. México: Ediciones UNAM, 1985: 53-99.

19. Bloor D. El programa fuerte en la sociología del conocimiento. En: Olivé L, (comp.) La explicación social del conocimiento. México: Ediciones UNAM, 1985: 100-128.

20. James W. Pragmatismo. Buenos Aires: Agentar; 1989.

Recibido: 12 de julio de 2006.

Aceptado: 3 de octubre de 2006. 